\title{
Isolation and characterisation of peroxiredoxin 6 promoter from sheep (Ovis aries)
}

\author{
Nan-Nan Liu ${ }^{1}$, Zeng-Shan Liu ${ }^{1}$, Shi-Ying Lu${ }^{1}$, Pan $\mathrm{Hu}^{1}$, \\ Ying Zhang ${ }^{1}$, Bao-Quan $\mathrm{Fu}^{2}$, Yan-Song $\mathrm{Li}^{1}$, \\ Yu Zhou ${ }^{1}$, Yu Zhang ${ }^{3}$, Hong-Lin Ren ${ }^{1}$ \\ ${ }^{1}$ Key Laboratory of Zoonosis Research, Ministry of Education, Institute of Zoonosis/College of Veterinary Medicine, \\ College of Animal Sciences, Jilin University, Xi An Da Lu 5333, Changchun 130062, China \\ ${ }^{2}$ State Key Laboratory of Veterinary Etiological Biology, \\ Key Laboratory of Veterinary Public Health of the Ministry of Agriculture, \\ Key Laboratory of Veterinary Parasitology of Gansu Province, \\ Lanzhou Veterinary Research Institute, Chinese Academy of Agricultural Sciences, Lanzhou 730046, China \\ ${ }^{3}$ Fifth Department of Orthopaedics, First Central Hospital of Baoding, Baoding 071000, China \\ renhl@jlu.edu.cn
}

Received: March 25, $2016 \quad$ Accepted: September 2, 2016

\begin{abstract}
Introduction: Peroxiredoxin $6(\operatorname{Prdx} 6)$ is a bifunctional protein and a unique 1-Cys $\operatorname{Prdx}$ of the peroxiredoxin family. The expression and regulation of Prdx6 are implicated in numerous physiological and pathological processes. Material and Methods: Eight stepwise truncated DNA fragments obtained from the 5'-flank region of the Prdx6 gene were prepared and subcloned into the pSEAP2-Enhancer vectors. To investigate the transcriptional activity of the truncated DNA fragments, the recombinant plasmids were transfected into the COS-1 cells and the transcriptional activity was measured via assaying the expression of the reporter gene of the secreted alkaline phosphatase. Results: A $3.4 \mathrm{~kb} 5$ '-upstream flank region of the Prdx6 gene was cloned and sequenced. The region from $-108 \mathrm{nt}$ to $-36 \mathrm{nt}$ of the $5^{\prime}$-flanking region of the $\operatorname{Prdx} 6$ gene contained basal transcriptional activity. Conclusion: This result provides the basis for further studies on the gene regulation of the Prdx6mediated biological processes and on screening for the transacting factors that interact with cis-acting elements of the Prdx6 gene promoter.
\end{abstract}

Keywords: Ovis aries, peroxiredoxin 6, promoter, transcriptional activity.

\section{Introduction}

Peroxiredoxin $6(\operatorname{Prdx} 6)$ is a bifunctional protein possessing both glutathione peroxidase and phospholipase $\mathrm{A}_{2}$ activities, and playing a major role in protection against oxidation (13), DNA damage $(5,7)$, and phospholipid metabolism (8). Prdx6 can be regulated by many factors. Oxidative stress can change the expression patterns of Prdx6 gene (10). For instance, $\mathrm{H}_{2} \mathrm{O}_{2}$ increased Prdx6 mRNA transcriptional levels in A549 cells (3). In addition, Prdx6 was downregulated in disk abalones challenged with viral haemorrhagic septicaemia virus (VHSV) (9). In contrast, the Prdx6 mRNA in the liver of Oplegnathus fasciatus can be up-regulated by synthetic polyinosinic:polycytidylicacid (poly I:C) and iridovirus (5). Prdx6 can also be regulated during bacterial invasion, and it is considered to be potentially relative to host responses against the invading bacteria (7). The level of Prdx6 was up-regulated in the liver and spleen of Scophthalmus maximus challenged by bacterial pathogens and poly I:C (16), and in marrow-derived macrophages stimulated by LPS and IFN- $\gamma$ (1). The environmental factors are also able to influence the expression of Prdx6 gene in organisms. As the pollution levels increase in marine surroundings, the expression of Prdx6 gene was highly up-regulated in Crassostrea gigas (4). Temperature stresses including low and high temperatures can also regulate the transcripts level of Prdx6 (12). 
The full-length cDNA of the Prdx6 gene from Ovis aries (OaPrdx6) was identified and analysed for the first time by our group (7). The OaPrdx6 mRNA is composed of a 5'-untranslated region (UTR) of $93 \mathrm{bp}$, a 3'-UTR of 985 bp with a poly (A) tail, and an open reading frame (ORF) of $675 \mathrm{bp}$ encoding a $25.1 \mathrm{kDa}$ protein of 224 amino acid residues. The expression profiles of the OaPrdx6 in the white blood cells of sheep challenged with Brucella are changed. Compared with the Brucella vaccine strain S2, the virulent field strain of Brucella melitensis can up-regulate the mRNA transcription level of OaPrdx6 (7).

The expression and regulation of Prdx6 are implicated in numerous physiological and pathological processes. Antioxidant-response elements (AREs) are considered to be the key regulators of Prdx6 gene of the basal transcription and the induced responses to oxidative stress (3). Nevertheless, the regulatory mechanisms for variabilities of Prdx6 expression underlying different responses remain unknown.

In the present study, we determined the transcriptional activity of the 5 '-upstream flank region of the sheep Prdx6 gene and provided the basis for further studies on regulation of Prdx6.

\section{Material and Methods}

Cells. The COS-1 cell line, Escherichia coli DH5a competent cells, was stored in Key Laboratory of Zoonosis Research, Ministry of Education, Jilin University (China).

Cloning of 5'-flanking region of OaPrdx6 gene. The genomic DNA (gDNA) was extracted from sheep (Ovis aries) lung according to the manufacturer's instructions by means of the TaKaRa MiniBEST Universal Genomic DNA Extraction Kit Ver. 5.0 (TaKaRa, Japan). A set of primers listed in Table 1 (GenQCS and GenQCA) were designed according to the genome DNA sequence (GenBank accession number: NW_004080175.1) and the full-length cDNA sequence of OaPrdx6 (GenBank accession number: KC342239). The 5'-flanking region of Prdx6 gene from the sheep (OaPrdx6) was cloned using the HiFi HotStart ReadyMix PCR Kit (KAPA, USA) with the extracted genomic DNA as the template. The PCR amplifications were performed following the standard PCR protocol provided in the kit. The conditions were as follows: $95^{\circ} \mathrm{C}$ for $5 \mathrm{~min} ; 28$ cycles of denaturation for $30 \mathrm{~s}$ at $98^{\circ} \mathrm{C}$, annealing for $30 \mathrm{~s}$ at $67^{\circ} \mathrm{C}, 3 \mathrm{~min}$ extension at $72^{\circ} \mathrm{C}$; then extension at $72^{\circ} \mathrm{C}$ for $5 \mathrm{~min}$. The PCR products were purified using the Axyprep PCR cleanup kit (Axygen, China), and then ligated into the pMD18-T vectors after 3'-dA-tailed. Finally, the recombinant plasmids were transformed into the $E$. coli DH5 $\alpha$ cells and sequenced by Comate Bioscience (China).

Stepwise truncation and transcriptional activity assay. Eight truncated DNA fragments of the 5 '-flanking region of the $\operatorname{OaPrdx} 6$ gene were cloned using PCR with specific primers (Tables 1 and 2). The conditions were as follows: $95^{\circ} \mathrm{C}$ for $5 \mathrm{~min}$; 30 cycles of denaturation for $30 \mathrm{~s}$ at $98^{\circ} \mathrm{C}$, annealing for $30 \mathrm{~s}$ at $65^{\circ} \mathrm{C}$, extension at $72^{\circ} \mathrm{C}$ for $90 \mathrm{~s}$; then extension at $72^{\circ} \mathrm{C}$ for $5 \mathrm{~min}$. The PCR products were subcloned into pSEAP2-Enhancer vector plasmids (Clontech, USA) digested with $K p n$ I and $B g l$ II restriction enzymes to construct the recombinant plasmids of the stepwise truncation (Table 2). The recombinant plasmids were transformed into E. coli DH5 $\alpha$ cells and sequenced by Shanghai Sangon Biological Engineering Technology \& Service (China). The recombinant plasmids containing different truncated DNA fragments were extracted without endotoxin from $E$. coli DH5 $\alpha$ cells using the Pure Yield ${ }^{\mathrm{TM}}$ Plasmid Miniprep System (Promega, USA) according to the manufacturer's protocol. The concentrations of plasmids were determined by the microplate spectrophotometer (Epoch, BioTek, USA).

The COS-1 cells were cultured in DMEM/F12 medium supplemented with $100 \mathrm{U} / \mathrm{mL}$ of penicillin, $100 \mu \mathrm{g} / \mathrm{mL}$ of streptomycin, and $10 \%$ foetal bovine serum (FBS), and seeded in triplicates into 96 microwell plates at a density of $2.0 \times 10^{4}$ cells/well. Twentyfour hours later, the medium was changed to fresh DMEM/F12 medium with $10 \%$ FBS and without penicillin and streptomycin. The pCMV-EGFP recombinant plasmids (constructed by our lab, as shown in Fig. 1) used as control and the pSEAP2Enhancer vector plasmids used as blank were transiently co-transfected by FuGENE $^{\circledR}$ HD Transfection Reagent (Promega, USA) according to the manufacturer's protocol.

Table 1. The sequences of primers used in the study

\begin{tabular}{lll}
\hline Object & Primer names & Sequences $\left(5^{\prime}\right.$-3') \\
\hline 5'-flanking gDNA clone & & \\
& GenQCS & 5'-CGGGGTACCTGGGCCATATCATGGCTTCTGCTTAC-3' \\
Stepwise truncation & 5'-GGA & \\
& JDAGATCTGGATGCGGCCGATGGTAGTATTTGC-3' & \\
& JDS119Q & 5'-GGAAGATCTGGCGGCAGCAGTGACGCGAGGAG-3' \\
& JDS191Q & 5'-CGGGGTACCGCAAACGACCTGGCCCCGCTTCC -3' \\
& JDS251Q & 5'-CGGGGTACCTCCAGACTCTCGCCCGGGTCGCC-3' \\
& JDS328Q & 5'-CGGGGTACCCAGGGCAGAGGCCTGGCGGGGTG-3' \\
& JDS499Q & 5'-CGGGGTACCCCGCGGATTCTGGCGGCTCCGTAGGGC-3' \\
& JDS601Q & 5'-CGGGGTACCGCGGTCAGGATTCCAGACGCTCACG-3' \\
& JDS1351Q & 5'-CGGGGTACCTGAGCAGACCATCCAGTCCAAGTAGC-3' \\
& JDS1946Q & 5'-CGGGGTACCTTCATTCCCAGCAGAAAATGGACAAG-3' \\
\hline
\end{tabular}

The letters underlined in the primer sequences stand for the restriction sites of $K p n$ I (GGTACC) and Bgl II (AGATCT) 
Table 2. The construction of stepwise truncation plasmids

\begin{tabular}{lllll}
\hline $\begin{array}{l}\text { Stepwise } \\
\text { truncation name }\end{array}$ & Primer names & Position & $\begin{array}{l}\text { Amplicon length } \\
\text { (bp) }\end{array}$ & $\begin{array}{l}\text { Recombinant } \\
\text { plasmids }\end{array}$ \\
\hline JD119 & $\begin{array}{l}\text { JDS119Q } \\
\text { JDA0Q } \\
\text { JDS191Q }\end{array}$ & $-36 \mathrm{nt}$ to $+83 \mathrm{nt}$ & 119 & EH-JD-36/+83 \\
JD191 & $\begin{array}{l}\text { JDA0Q } \\
\text { JDS251Q } \\
\text { JDA0Q }\end{array}$ & $-108 \mathrm{nt}$ to $+83 \mathrm{nt}$ & 191 & EH-JD-108/+83 \\
JD251 & $\begin{array}{l}\text { JDS328Q } \\
\text { JDA0Q } \\
\text { JDS499Q }\end{array}$ & $-245 \mathrm{nt}$ to $+83 \mathrm{nt}$ & 328 & EH-JD-168/+83 \\
JD328 & $\begin{array}{l}\text { JDA0Q } \\
\text { JDS601Q } \\
\text { JDA0Q }\end{array}$ & $-416 \mathrm{nt}$ to $+83 \mathrm{nt}$ & 499 & EH-JD-245/+83 \\
JD499 & $\begin{array}{l}\text { JDS1351Q } \\
\text { JDA0Q } \\
\text { JD61946Q }\end{array}$ & $-518 \mathrm{nt}$ to $+83 \mathrm{nt}$ & 601 & EH-JD-416/+83 \\
JD1351 & $-1268 \mathrm{nt}$ to $+83 \mathrm{nt}$ & 1351 & EH-JD-518/+83 \\
JD1946 & $-1863 \mathrm{nt}$ to $+83 \mathrm{nt}$ & 1946 & EH-JD-1268/+83 \\
\hline
\end{tabular}
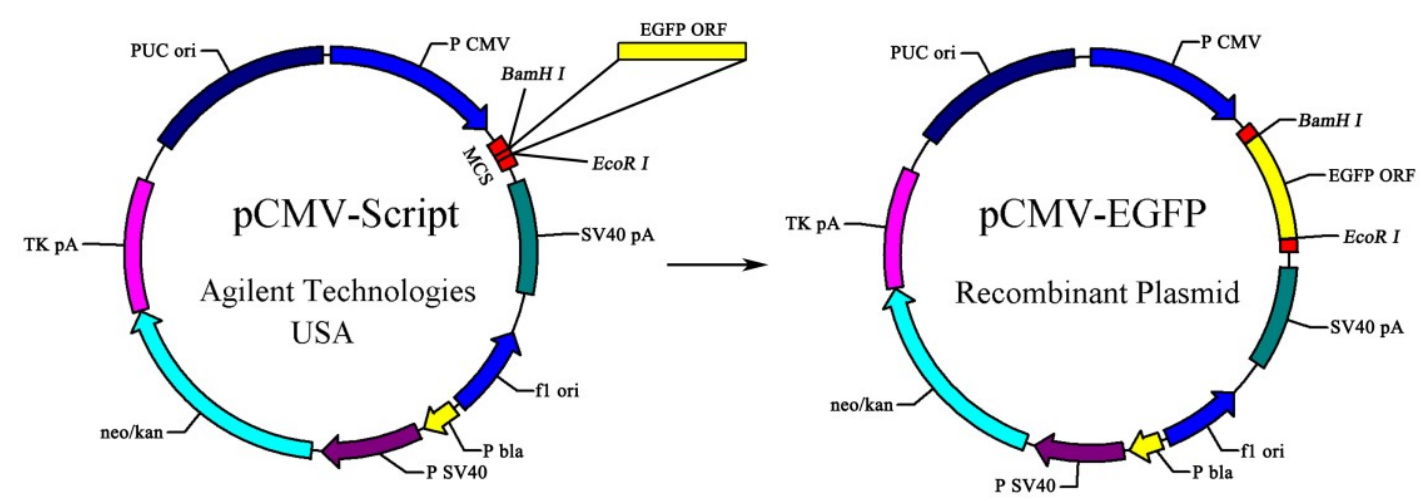

Fig. 1. Schematic representation of the construction strategy of the pCMV-EGFP recombinant plasmid. EGFP - enhanced green fluorescent protein; OFR - open reading frame

Forty-eight hours after transfection, the quantification of the secreted alkaline phosphatase (SEAP) activity in culture supernatant of the transfected cells was determined by SEAP Reporter Gene Assay chemiluminescent kit (Promega, USA) following the protocol provided by the manufacturer. The green fluorescence signals of cells were detected using the Infinite ${ }^{\circledR}$ M1000 PRO-multimode microplate reader (TECAN, Switzerland). The value of SEAP in each sample was normalised by the fluorescent values of the expressed green fluorescent proteins derived from the pCMV-EGFP plasmids in cells. The results were calculated by the relative quantities regarding the relative values of SEAP from the pSEAP2-Enhancer plasmid group as a negative control. The transcriptional activity assay was conducted in triplicates. Significant differences were determined by one-way analysis of variance (ANOVA) using the SPSS 19 software.

\section{Results}

The 5' flanking region of the sheep Prdx6 gene. The genomic DNA of Ovis aries was extracted from the lungs and a $3.4 \mathrm{~kb} 5$ '-flanking region of the
OaPrdx6 gene was cloned and sequenced. The sequence of 5'-flanking region was basically consistent with the original sequence (GenBank accession number: NW_004080175.1), deposited in GenBank and assigned the accession number KM459018. The partial nucleotide sequence of the 5 '-flanking region of the sheep Prdx6 gene is shown in Fig. 2.

The transcription factor binding sites (TFBSs) in the 5'-flanking region of OaPrdx6 genomic DNA were predicted, and included binding sites for the following transcription factors: Sp-1, Egr-1, RXR- $\alpha$, USF, PU.1, YY1, GATA-1, c-Ets-1, CPE binding, CRE-BP1, HB, Pit-1a, TBP, RelA, NF-kapaB, AGIE-BP1, Oct-1, ISGF-3, ICSBP, c-Rel, MBP-2, AP-2- $\alpha$, NF-1, AP-2, REV-ErbA, CREB, SRF, C/EBP- $\alpha$, C/EBP- $\beta$, GR, and RAR- $\alpha$, in different quantity, as shown in Fig. 2 . The TFBS for $\mathrm{Sp}-1$ was the most abundant and widely distributed in 5'-flanking region of $\operatorname{OaPrdx} 6$ gene, especially for the region from -245 to +83 (Figs 2 and 3).

Stepwise truncation of the 5 ' flanking regions of the OaPrdx6 gene. Eight truncated DNA fragments of the 5'-flanking region of OaPrdx6 gene were cloned with the predicted length of $119 \mathrm{bp}, 191 \mathrm{bp}, 251 \mathrm{bp}$, $328 \mathrm{bp}, 499 \mathrm{bp}, 601 \mathrm{bp}, 1351 \mathrm{bp}$, and $1946 \mathrm{bp}$, and then subcloned into the pSEAP2-Enhancer vectors to 
construct eight recombinant plasmids (Table 2), which were respectively transformed into $E$. coli $\mathrm{DH} 5 \alpha$. The PCR products were visualised in $1 \%$ agarose gel stained with EB (Fig. 4). These recombinant plasmids were confirmed by DNA sequencing. The sequencing results showed that the nucleotide sequences of eight DNA fragments were consistent with the nucleotide sequence of the $5^{\prime}$-flanking region of $\operatorname{OaPrdx} 6$ gene (GenBank accession number KM459018).
101

102

201

301

401

501

601

701

801

901

1101

1201

1301

1401

1501

1601

1701

1801

1901

2001
$-1863$

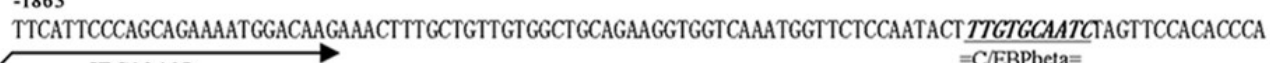
JDS1946Q

TTATGTCTAACAGGTCACAAACATTTATCAATAAAATATGGTCTAACCTTTAGGAGTTCACAATCGTAATGTACTGTTGTTCTTCCAAACGGCCATTGCC $=$ RAR-alpha $=$ $=$ REV $\cdot$ ErbA $=$

AAACCCTTCACTTTTTCCTTCCTACCTTTTAACTCTTAACAATTTGGCATCCTGCOCT CCCCCAACCCTCATCAGCATTTACTGTTTTTATCGTAACTG

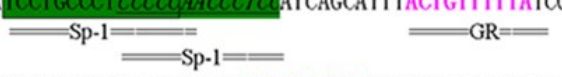

TATAGTATATCTGCTTTCCTCCTAAGAGGAAAGACTCTCTCTTCTGTATCACCAGGCCTAGCATAGCACATAGAACACAGTCAGCAAAGCAAGGGTTATT $=\mathrm{NF} \cdot \mathrm{kapaB}=$ $=\mathrm{C} / \mathrm{EBPalpha}=$

GCATAAACTACTATCATCCAAGTAATAGCTACCATTTCCAGAGCACTT CTATGTAAACTACTAAGATAGTTACGAAAGCAGATGCACCTGACAATTGGT $\mathrm{C} / \mathrm{EBPbeta}=\quad \quad=\mathrm{Oct}-1=\quad-1268$ ATTACT GGCCTGGAGGTTGAGGGTCTTATCCCTTGGTGCACTATGGCAGAGGTTAACTACTTACAGTTTATCTACACTATCCTGGAGGCTTTCCCTGAGC AGACCATCCAGTCCAAGTAGCACCTGTTACCCTCCAGCACATGCTACTGTCTAATATATTCCTATTTAATGATGTCTCCCTTACTATAATGAAAGCTCCA $\longrightarrow \mathrm{JDS} 1351 \mathrm{C}=\mathrm{SRF}=\Longrightarrow \mathrm{CREB}=$

AGAGAACAGTCATCTTGCCTAACTTGGGCTTGGCAGGTAGAAAGTTAAATAAATGAATCTAAACTTCCATTTCTTTGGGTGGCCATGTTGACTTGCACAC

TGTAGATTACAAGTAGGTTTTTATACTACTACTACTACTAAGTCACTTCAGTTGTGTCCGACTCTGTGTGACCCCATAGACAGCAGCCCACCAGGCTCCO $=$ REV-ErbA $=\overline{A P} \cdot 2==$ AP-2alpha $=$

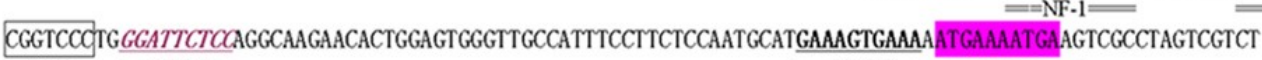

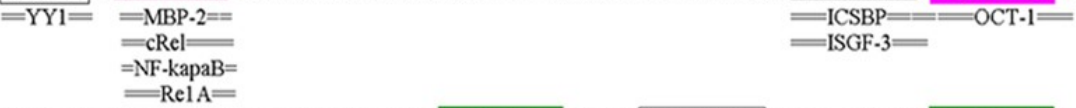

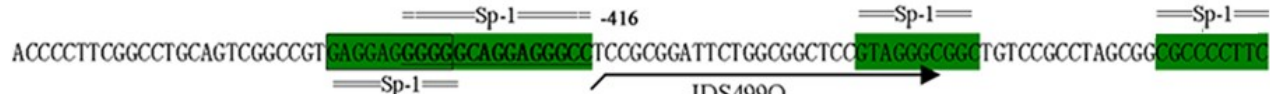

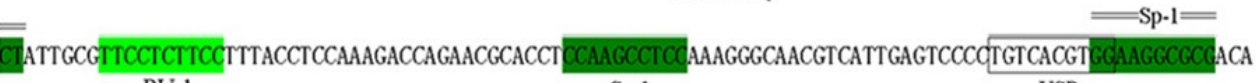
$=\mathrm{PU} .1=$

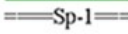

$\mathrm{USP}=$

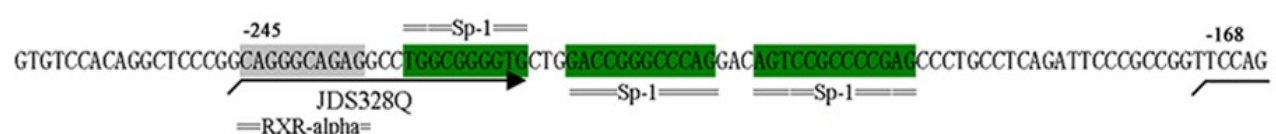

$=$ RXR-alpha

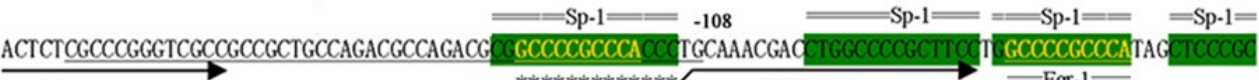
$*$ * J

=s-GC-box

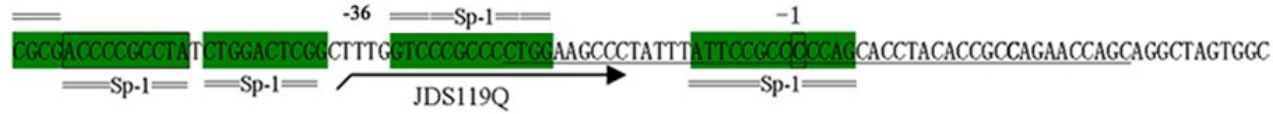

Start codon AGTCGGTGCTGCACCTCTTCTTACTCCTCGCGTCA TTGCTGCCGCCATGCCCGGAGGTCTCCTCCTCGGGGACGAGGCTCCCAACTTCGAGGCAAATACT ACCATCGGCCGCATCC

JDA0Q $\underline{\mathrm{S}} \mathrm{S} \cdot \mathrm{l}=$

Fig. 2. The partial nucleotide sequence of the 5'-upstream region of the sheep Prdx6 gene. The numbers represent the nucleotide position relative to the newly identified transcription initiation site $(+1)$. The predicted cis-elements and the primers used for the construction of stepwise truncation are marked 
Transcriptional activity analyses of the truncated 5' flanking regions of the OaPrdx6 gene. The recombinant plasmids containing the truncated 5 '-flanking region of $\operatorname{OaPrdx} 6$ gene were transferred into COS-1 cells. Forty-eight hours after transfection, the cell culture supernatant was collected and the SEAP Reporter Gene Assay chemiluminescent kit was used to analyse the transcriptional activity. The results showed that the recombinant plasmids with six different DNA fragments (EH-JD-108/+83, EH-JD-168/+83, EH-JD245/+83, EH-JD-416/+83, EH-JD-518/+83, and EHJD-1268/+83) had higher transcriptional activity compared with the empty pSEAP2-Enhancer vectors and recombinant plasmids with the other two different DNA fragments (EH-JD-36/+83 and EH-JD-1863/+83) (Fig. 5). A decrease in transcriptional activity was observed when the deletion was extended to $-36 \mathrm{nt}$ (Fig. 5). This result suggests that a basal promoter region lies in $-108 \mathrm{nt}$ to $-36 \mathrm{nt}$ region. The transfection of the EH-JD-1268/+83 resulted in a conspicuous transcriptional activity while the transfection of the EH-JD-1863/+83 resulted in no more transcriptional activity than the empty pSEAP2-Enhancer vectors (Fig. 5). This result implies the presence of negative regulatory elements in the $-1268 \mathrm{nt}$ to $-1863 \mathrm{nt}$.

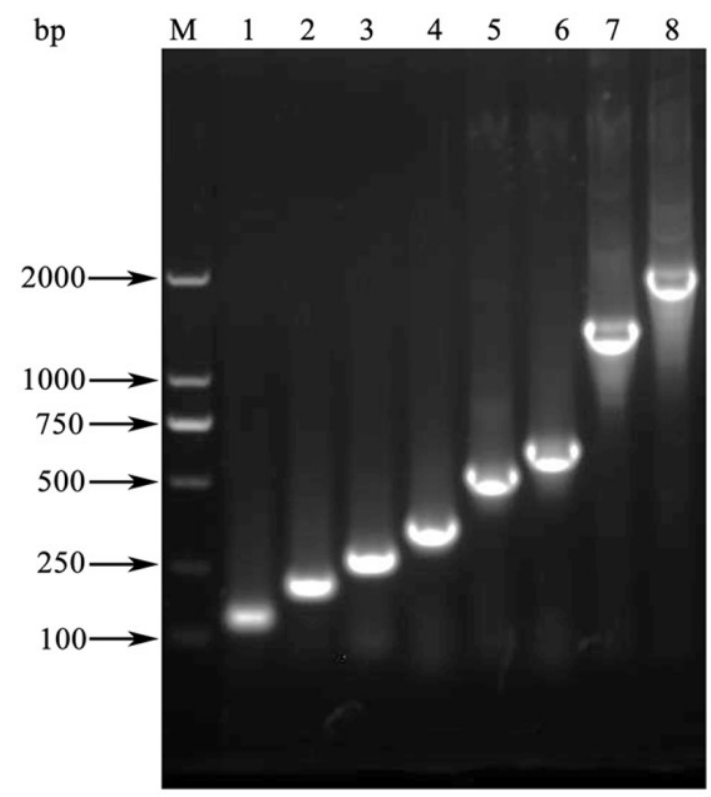

Fig. 4. PCR amplification results of the stepwise truncated OaPrdx6 5'-flanking regions. M - DL2000 DNA Marker (TaKaRa, Japan); 1-8 - the fragments amplified using the same antisense primer (JDA0Q) and the sequential sense primers (JDS119Q, JDS191Q, JDS251Q, JDS328Q, JDS499Q, JDS601Q, JDS1351Q, and JDS1946Q)

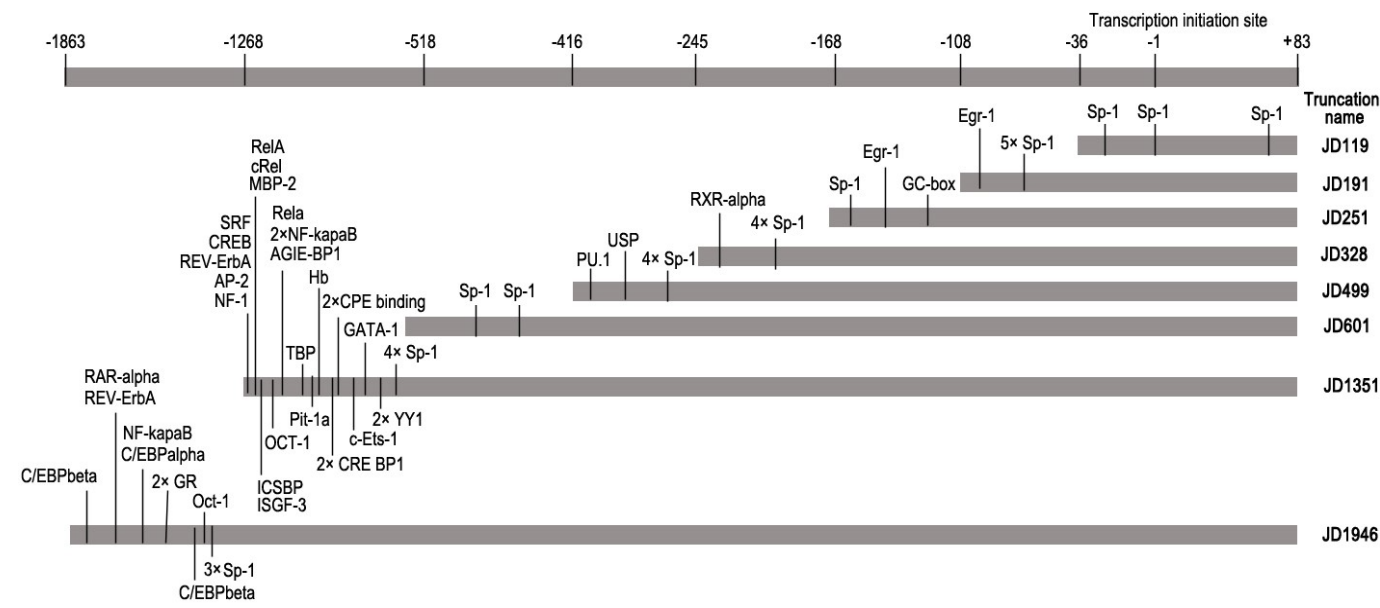

Fig. 3. Schematic representation of putative transcription factor binding sites in the promoter of $\operatorname{Prdx} 6$ gene $(-1863$ to +83$)$

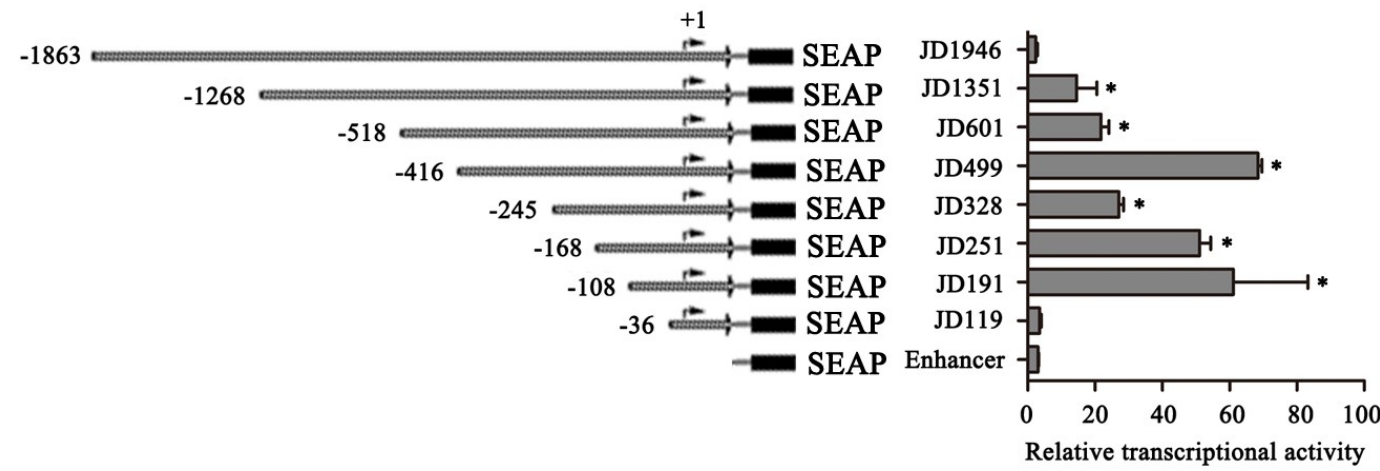

Fig. 5. Transcriptional activity assay of the stepwise truncated OaPrdx 65 '-flanking regions. The left side - schematic representation of recombinant constructs bearing the SEAP reporter gene fused to various OaPrdx6 5 -flanking regions. Numbers represent the nucleotide position relative to the newly identified transcription initiation site; a curved arrow indicates the transcription initiation site. The right side - relative transcriptional activity of various stepwise truncated OaPrdx6 5'-flanking regions. The results were calculated based on the relative quantification, with the transcriptional activity of pSEAP2-Enhancer empty vector as a value of $100 \%$. Data are presented as the mean relative expression \pm SD from transcriptional activity assay performed in triplicate. An asterisk indicates the statistically significant difference $V s$ pSEAP2-Enhancer empty vector group $(\mathrm{P}<0.05)$ 


\section{Discussion}

Prdx6 is a protease with peroxidase and phospholipase $\mathrm{A}_{2}$ activities. Multiple pathophysiological factors can cause diverse changes in the content of Prdx6 gene. On the other hand, several studies demonstrated that the alteration of Prdx6 gene expression plays a vital role in some disease states, for instance over-expression of Prdx6 can promote tumour growth in lung cancer (15). In our previous studies, we found that brucellae with different virulence have a different effect on regulation of OaPrdx6 expression (7), but the molecular mechanisms involved in the regulation remained unknown.

In the present study, we identified and characterised the sheep Prdx6 gene promoter. A CpGisland, which was associated with the transcription initiation of many genes (6), was found in the $5^{\prime}$ flanking region of the OaPrdx6 gene. The CpGisland was $598 \mathrm{bp}$ from $-517 \mathrm{nt}$ to $+81 \mathrm{nt}$ containing a s-GC-box marked by asterisks in Fig. 2. However, we did not find the classic TATA-box in 5 ' flanking region of $\operatorname{OaPrdx} 6$ gene, and this result was consistent with the previous report (14). There were two core promoters (CP) predicted and named as $\mathrm{CP} 1$ : ${ }^{1839}$ CTGGAAGCCCTATTTATTCCGCCCCCAGCAC CTACACCGCCAGAACCAGC ${ }^{1889}$ and CP2: ${ }^{1706} \mathrm{CGC}$ CCGGGTCGCCGCCGCTGCCAGACGCCAGACGC GGCCCCGCCCACCCT $^{1756}$ shown in Fig. 2. To identify the basal promoter region, the $5^{\prime}$ flanking region of $\operatorname{OaPrdx} 6$ gene was studied by the DNA truncation and the transcriptional activity analyses. The results showed that JD191 truncated DNA fragment has a much higher transcriptional activity than JD119 truncated DNA fragment. The JD119 truncated DNA fragment containing three TFBS for Sp-1 and CP1 core promoter showed no transcriptional activity, suggesting that the presence of TFBS for Sp-1 downstream of CP1 had no effect on transcriptional activity of the CP1. However, the JD191 truncated DNA fragments containing five more TFBS for Sp-1 and one TFBS for Egr-1 showed high transcriptional activity. So the presence of TFBS for Egr-1 may have a positive effect on transcriptional activity.

$\mathrm{Sp}-1$ is an oxidative stress-induced transcription factor (11) that can bind Sp1-responsive elements and increase transcription of $\operatorname{Prdx} 6$ gene (2). TFBSs for Sp-1 were ubiquitous in 5'-proximal region of Prdx6 gene promoter. The high content of TFBSs for Sp-1 was consistent with the antioxidant activity of Prdx6. In the present study, the TFBS for Sp-1 did not show the ability to increase transcriptional activity of $\operatorname{Prd} d x 6$ gene promoter. The reason why the presence of TFBSs for Sp-1 did not have an effect on increasing the transcriptional activity of $\operatorname{Prd} d x 6$ gene promoter may be that no stimulation was used in this study to investigate the transcriptional activity of $\operatorname{Prdx} 6$ gene promoter. Thus, the regulation of transcriptional activity of $\operatorname{Prdx} 6$ gene promoter needs further investigation.
In summary, the 5'-flanking region sequence of OaPrdx6 gene was cloned and its transcriptional activity was identified. This result provided the basis for further studies on gene regulation of the Prdx6mediated biological processes, and on screening for the transacting factors interacting with cis-acting elements of the $\operatorname{Prdx} 6$ promoter.

Conflict of Interests Statement: The authors declare that there is no conflict of interests regarding the publication of this article.

Financial Disclosure Statement: This work was supported by the Open Fund of the State Key Laboratory of Veterinary Etiological Biology, Lanzhou Veterinary Research Institute, Chinese Academy of Agricultural Sciences (No. SKLVEB2015KFKT006); the Science \& Technology Development Project of Jilin Province, China (No. 20150204078NY); and the Project 2015041 Supported by Graduate Innovation Fund of Jilin University.

Animal Rights Statement: None required.

Acknowledgements: These authors (Nan-Nan Liu, Zeng-Sang Liu, Shi-Ying $\mathrm{Lu}$ and Pan $\mathrm{Hu}$ ) contributed equally to this study with Nan-Nan Liu and Zeng-Shan Liu. Hong-Lin Ren was the corresponding author. We do appreciate them for designing and composing this study.

\section{References}

1. Bast A., Erttmann S.F., Walther R.,Steinmetz I.: Influence of iNOS and COX on peroxiredoxin gene expression in primary macrophages. Free Radic Biol Med 2010, 49, 1881-1891.

2. Chhunchha B., Fatma N., Bhargavan B., Kubo E., Kumar A., Singh D.P.: Specificity protein, Sp1-mediated increased expression of Prdx6 as a curcumin-induced antioxidant defense in lens epithelial cells against oxidative stress. Cell Death Dis 2011, 2, e234.

3. Chowdhury I., Mo Y., Gao L., Kazi A., Fisher A.B., Feinstein S.I.: Oxidant stress stimulates expression of the human peroxiredoxin 6 gene by a transcriptional mechanism involving an antioxidant response element. Free Radic Biol Med 2009, 46, 146-153.

4. David E., Tanguy A., Moraga D.: Peroxiredoxin 6 gene: a new physiological and genetic indicator of multiple environmental stress response in Pacific oyster Crassostrea gigas. Aquat Toxicol 2007, 84, 389-398.

5. De Zoysa M., Ryu J.H., Chung H.C., Kim C.H., Nikapitiya C., Oh C., Kim H., Saranya Revathy K., Whang I., Lee J.: Molecular characterization, immune responses and DNA protection activity of rock bream (Oplegnathus fasciatus), peroxiredoxin 6 (Prx6). Fish Shellfish Immunol 2012, 33, 28-35.

6. Fatemi M., Pao M.M., Jeong S., Gal-Yam E.N., Egger G., Weisenberger D.J., Jones P.A.: Footprinting of mammalian promoters: use of a $\mathrm{CpG}$ DNA methyltransferase revealing nucleosome positions at a single molecule level. Nucleic Acids Res 2005, 33, e176.

7. Liu N.N., Liu Z.S., Lu S.Y., Hu P., Li Y.S., Feng X.L., Zhang S.Y., Wang N., Meng Q.F., Yang Y.J., Tang F., Xu Y.M., Zhang W.H., Guo X., Chen X.F., Zhou Y., Ren H.L.: Full-length 
cDNA cloning, molecular characterization and differential expression analysis of peroxiredoxin 6 from Ovis aries. Vet Immunol Immunopathol 2015, 164, 208-219.

8. Manevich Y., Fisher A.B.: Peroxiredoxin 6, a 1-Cys peroxiredoxin, functions in antioxidant defense and lung phospholipid metabolism. Free Radic Biol Med 2005, 38, 1422-1432.

9. Nikapitiya C., De Zoysa M., Whang I., Kim C.G., Lee Y.H., Kim S.J., Lee J.: Molecular cloning, characterization and expression analysis of peroxiredoxin 6 from disk abalone Haliotis discus discus and the antioxidant activity of its recombinant protein. Fish Shellfish Immunol 2009, 27, 239-249.

10. Pak J.H., Kim T.I., Joon Kim M., Yong Kim J., Choi H.J., Kim S.A., Tchah H.: Reduced expression of 1-cys peroxiredoxin in oxidative stress-induced cataracts. Exp Eye Res 2006, 82, 899-906.

11. Ryu H., Lee J., Zaman K., Kubilis J., Ferrante R.J., Ross B.D., Neve R., Ratan R.R.: Sp1 and Sp3 are oxidative stress-inducible, antideath transcription factors in cortical neurons. J Neurosci 2003, 23, 3597-606.
12. Wang Q., Chen K., Yao Q., Zhao Y., Li Y., Shen H., Mu R.: Identification and characterization of a novel 1-Cys peroxiredoxin from silkworm, Bombyx mori. Comp Biochem Physiol B Biochem Mol Biol 2008, 149, 176-182.

13. Wang Y., Phelan S.A., Manevich Y., Feinstein S.I., Fisher A.B.: Transgenic mice overexpressing peroxiredoxin 6 show increased resistance to lung injury in hyperoxia. Am J Respir Cell Mol Biol 2006, 34, 481-486.

14. Wu X., Ji P., Zhang L., Bu G., Gu H., Wang X., Xiong Y., Zuo B.: The expression of porcine Prdx6 gene is up-regulated by C/EBPbeta and CREB. PLoS One 2015, 10, e0144851.

15. Yun H.M., Park K.R., Lee H.P., Lee D.H., Jo M., Shin D.H., Yoon D.Y., Han S.B., Hong J.T.: PRDX6 promotes lung tumor progression via its GPx and iPLA2 activities. Free Radic Biol Med 2014, 69, 367-376.

16. Zheng W.J., Hu Y.H., Zhang M., Sun L.: Analysis of the expression and antioxidative property of a peroxiredoxin 6 from Scophthalmus maximus. Fish Shellfish Immunol 2010, 29, 305-311. 